New perimetric

threshold test algorithm

with dynamic strategy

and tendency oriented

perimetry (TOP) in

\section{glaucomatous eyes}

\begin{abstract}
Purpose To investigate the time-wise reliability and efficiency of two new perimetric test algorithms, two computerised static threshold perimetry strategies, namely dynamic strategy (DS) and tendency oriented perimetry (TOP), were compared with the standard full-threshold strategy (normal strategy, NS).

Methods We examined 41 eyes of 41 normal individuals without any ocular disease and 36 eyes of 36 glaucomatous patients, with the NS (4-to-2 dB), DS and TOP using an Octopus 1-2-3 perimeter. We analysed test time, stimulus time and the two global indices, mean sensitivity (MS) and loss variance (LV). Program 32X was used as test grid pattern. Results The mean test time for the NS was reduced by $52 \%$ with the DS and by $78 \%$ with the TOP strategy. Concerning the global indices, the MS value did not differ among the three strategies in the control or glaucoma group. However, the LV value was lower in the TOP strategy compared with the other two strategies in the glaucoma group. This suggested that the TOP strategy underestimated local glaucomatous visual field defects. The ability to detect early-stage glaucoma with the DS and TOP was inferior to that with the NS. Conclusions The DS was more efficient than the TOP strategy for the detection of early glaucomatous defects, whereas the TOP strategy required less testing time. The TOP strategy may be an appropriate approach for patients in whom time-consuming perimetry is not possible, or in whom the visual field defect is already advanced.
\end{abstract}

Key words Dynamic strategy, Full-threshold strategy, Glaucomatous eye, Perimetric test, Tendency oriented perimetry (TOP)

At present, perimetry mainly using a static automatic perimeter is an indispensable examination in daily ophthalmological
HIDETAKA MAEDA, MAKOTO NAKAURA, AKIRA NEGI

practice. ${ }^{1-3}$ Measurement of the threshold by the 4-to-2 dB up-down strategy, ${ }^{4,5}$ which is a standard method used in automated perimetry, allows accurate measurement of differential light sensitivity but imposes a significant test time burden. ${ }^{6}$ Therefore, more time-efficient methods of measuring threshold are required. In recent years, some new algorithms have been proposed for the examination of the threshold, markedly shortening measurement time. The dynamic strategy (DS) ${ }^{7,8}$ is a program developed by Weber and Klimaschka, ${ }^{9}$ who noted the flatter slope of the frequency of the seeing curve in areas of low visual sensitivity and attempted to shorten the measurement time by changing the step size used for the measurement of the threshold in defect depth ranges of $2-10 \mathrm{~dB}$. The tendency oriented program (TOP) strategy $^{10}$ was developed by de la Rosa et al., ${ }^{11}$ who noted the similarity of the threshold between spatially oriented neighbouring measurement points and evaluated the threshold tendency based on the response at each point to a single stimulus presentation. We performed perimetry by the DS and TOP strategy using an Octopus perimeter in patients with glaucoma, and compared the results with those obtained by the conventional full-threshold method.

\section{Subjects and methods}

The subjects consisted of 41 eyes of 41 normal adult volunteers and 36 eyes of 36 patients with glaucoma under follow-up at our department, from whom informed consent for this study was obtained. The normal control group had no ophthalmological abnormalities except slight ametropia. The glaucoma group had visual field defects corresponding to glaucomatous change at the optic disc, and their intraocular pressures were controlled below $25 \mathrm{mmHg}$ with topical medication only. No subject was on miotic agents which could influence the pupil size. Their visual field defects were classified by the

\author{
H. Maeda \\ M. Nakaura \\ A. Negi \\ Kobe University \\ Kobe, Japan
}

Hidetaka Maeda, MD

Department of

Ophthalmology

Kobe University School of

Medicine

Chuo-ku, Kobe-shi

Hyogo 650, Japan

Tel: +8178 3826048

Fax: +8178 3826059

e-mail:

hidetaka@med.kobe-u.ac.jp

Received: 5 August 1999 Accepted in revised form: 6 March 2000
7-5-2 Kusunoki-cho 
Table 1. The number of patients according to the Aulhorn-Greves classification stage

\begin{tabular}{llr}
\hline Normal control eyes: & 41 eyes of 41 subjects \\
Glaucomatous eyes: & 36 eyes of 36 patients \\
& Stage I & 18 patients \\
& Stage II & 10 patients \\
& Stage III & 8 patients \\
\hline
\end{tabular}

results of 'gold standard' full-threshold strategy. The visual field defects of the eyes in the glaucoma group were graded according to the Aulhorn-Greves' classification system. ${ }^{12}$ The visual field results of glaucomatous patients were within stage III or lower according to this classification. The number of subjects in each stage is shown in Table 1 . In all subjects, the refractive error was between $-5 \mathrm{D}$ and $+3 \mathrm{D}$, and the corrected visual acuity was at least $20 / 20$. To exclude the effects of opacity of the optic media or age-related changes on the visual field, the age of the subjects was limited to between 30 and 65 years, and patients with cataract were excluded. Patients with optic disc hypoplasia or tilted disc were also excluded. The clinical backgrounds of the normal control group and the glaucoma group are shown in Table 2. No significant difference was observed in any parameter except the intraocular pressure between the two groups (unpaired $t$-test).

The Octopus 1-2-3 perimeter was used for perimetric testing. Initially, perimetry was performed with the normal Octopus program 32 using the 4-to- $2 \mathrm{~dB}$ updown strategy (NS) by the conventional full-threshold method. Within 2 weeks after this measurement, the Octopus program 32 test was performed again by the same examiner in each subject using the DS and TOP strategy. The measurement conditions ${ }^{12-14}$ were as follows: target size III, $4\left(\mathrm{~mm}^{2}\right)$; background luminance, 31.6 (asb); target presentation time, 100 (ms); and maximum target luminance, 4000 (asb). The measurement order of the two strategies was random. Those patients who had had at least one Octopus 32 test within approximately 1 year and who had previous experience $^{14}$ of automated threshold perimetry were recruited. Thus, all subjects were familiar with automated threshold perimetry testing. For each strategy, the results obtained in the examination showing high reliability (false-positive responses, false-negative responses and poor fixation: $\leqslant 15 \%$ ) were adopted.

The measurement time, number of stimulus presentations, and mean sensitivity (MS) and the loss variance $(\mathrm{LV})$ as global indices, were compared among

Table 2. The clinical backgrounds of the normal control group and the glaucoma group

\begin{tabular}{lccc}
\hline & $\begin{array}{c}\text { Control } \\
\text { subjects }\end{array}$ & $\begin{array}{c}\text { Glaucoma } \\
\text { patients }\end{array}$ & $p$ value $^{\mathrm{a}}$ \\
\hline Cases & 41 & 36 & \\
Age (years) & $51.1 \pm 13.5$ & $53.7 \pm 10.5$ & 0.20 \\
Refraction (D) & $-2.17 \pm 1.38$ & $-3.08 \pm 1.79$ & 0.47 \\
IOP (mmHg) & $15.7 \pm 1.96$ & $18.7 \pm 1.34$ & 0.05 \\
Pupil diameter (mm) & $3.7 \pm 1.0$ & $3.6 \pm 1.1$ & 0.38 \\
\hline
\end{tabular}

${ }^{\text {a Unpaired } t \text {-test. }}$ the NS, DS and TOP strategy in both the glaucoma and normal control groups. The sensitivity and specificity of the DS and TOP strategy were also evaluated. Sensitivity and specificity results were analysed according to standard methods. The sensitivity of the DS and TOP strategy with Octopus automated perimetry was defined as the number of abnormal visual fields divided by the number of 'gold standard' NS abnormal visual fields expressed as a percentage. The specificity was determined by dividing the number of perimetry fields with the DS and TOP strategy by the number of the results of visual fields with NS expressed as a percentage. The confidence interval and the probability level in each statistical analysis were set at 95\% and 5\% for statistical significance.

\section{Results}

Comparison of the measurement time and the frequency of target presentation

Fig. 1 shows the measurement time using the NS, DS or TOP strategy. The mean measurement time using the NS method was $13 \mathrm{~min} 32 \mathrm{~s}$ (812 s) in the control group and $15 \mathrm{~min} 17 \mathrm{~s}$ (917 s) in the glaucoma group. The measurement time using the DS was $52.6 \%$ of that using the NS in the control group and $52.3 \%$ in the glaucoma group. Similarly, the mean number of stimulus presentations per test session using the NS was 351 in the control group and 372 in the glaucoma group. The group mean number of stimulus presentations for normal subjects using the DS and TOP strategy were $44 \%$ and $23 \%$ respectively of the group mean for the NS. Using the TOP strategy, the frequency of stimulation was consistent at 76 times in every subject for both control and glaucoma groups.

The measurement times in glaucoma patients for DS and TOP, as compared with NS, are shown in Table 3. Using the DS, measurement time was proportionately shorter in patients with more advanced visual field impairment. The TOP strategy always presented 76 stimuli, and the measurement time was also nearly

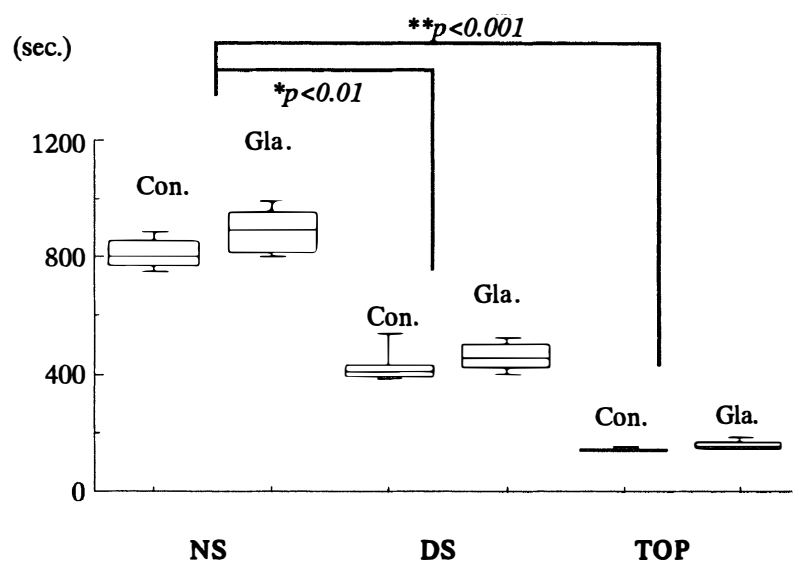

Fig. 1. Measurement time using the NS (normal strategy), DS (dynamic strategy) or TOP (tendency oriented programme) strategy. Con., normal control eyes; Gla., glaucoma eyes. p values are from an unpaired $\mathrm{t}$-test. 
Table 3. The measurement time according to the disease stage

\begin{tabular}{lrcc}
\hline & \multicolumn{4}{c}{ Measurement time (s) } \\
\cline { 2 - 4 } Aulhorn-Greves classification & NS & DS & TOP \\
\hline Stage I & $843.2 \pm 72.8$ & $455.4 \pm 72.8(45.2 \%)$ & $157.8 \pm 12.8(81.9 \%)$ \\
Stage II & $896.8 \pm 98.6$ & $487.2 \pm 56.6(46.7 \%)$ & $154.4 \pm 16.3(83.8 \%)$ \\
Stage III & $1056.6 \pm 106.7$ & $527.3 \pm 89.6(51.0 \%)$ & $155.2 \pm 13.8(85.4 \%)$ \\
\hline
\end{tabular}

Values in parentheses are the percentage shortening relative to NS.

NS, normal strategy; DS, dynamic strategy; TOP, tendency oriented perimetry.

consistent irrespective of the subjects; therefore, the percentage time reduction was more marked in patients with more advanced visual field loss.

\section{Comparison of global indices}

Mean sensitivity (MS)

The distribution of MS values in the control group is shown in Fig. 2. The MS value did not significantly differ among the NS, DS and TOP strategy in this group $(p=0.263$, one-way repeated measures ANOVA). Similarly, as shown in Fig. 2, no significant differences were observed in the MS value among three strategies in the glaucoma group $(p=0.322)$.

\section{Loss variance ( $L V)$}

The LV value did not differ significantly among the NS, DS and TOP strategies in the normal control group, as shown in Fig. 3. However, in the glaucoma group, significant differences were observed between NS and DS (unpaired $t$-test, $p<0.01$ ) and between NS and TOP $(p<0.001)$. In particular, the LV value was lower using the TOP strategy than using the other two strategies.

\section{Sensitivity and specificity}

The sensitivity and specificity of the DS and TOP strategy are shown in Table 4. Sensitivity tests showed that the DS detected 32 abnormal glaucomatous visual fields out of a

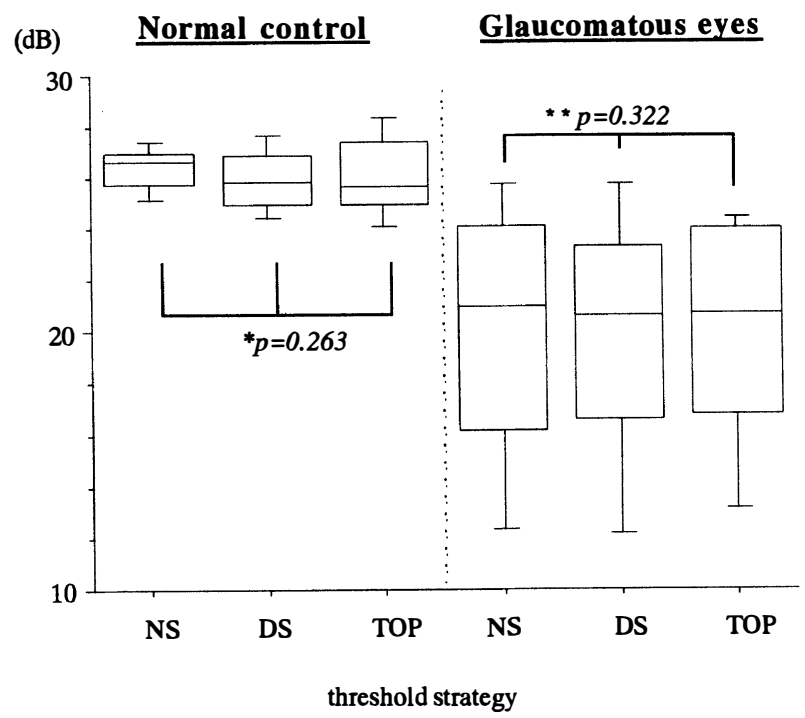

Fig. 2. The distribution of mean sensitivity (MS) values in the control group and the glaucoma group. total of 36 abnormal fields (88.9\%). The specificity of DS was $97.6 \%$ (40/41). Similarly, the sensitivity of the TOP strategy was $80.6 \%$, and its specificity was $90.2 \%$.

\section{Discussion}

Recent interest in static automatic perimetry for the diagnosis $^{1}$ and follow-up ${ }^{15,16}$ of glaucoma covers two aspects. One is early detection of glaucoma based on new aspects of visual function that completely differ from conventional perimetry. In this regard, blue on yellow perimetry, ${ }^{17}$ high-pass resolution perimetry ${ }^{18}$ and frequency doubling perimetry ${ }^{19}$ have been developed. The other is shortening the measurement time by improving the strategy while maintaining the advantages of the full-threshold method, ${ }^{5}$ an established method that has been frequently used. The latter strategy includes measurement methods using a glaucoma model represented by the Swedish interactive threshold algorithm (SITA) ${ }^{20}$ and the DS and TOP strategy used in this study. Many studies ${ }^{20-23}$ have been conducted on the SITA at various institutions. On the other hand, there have been only a few studies on the DS and TOP strategy in glaucoma patients. Therefore, we performed perimetry using the DS and TOP strategy in glaucoma patients and compared the results with those using the NS.

Normal control Glaucomatous eyes

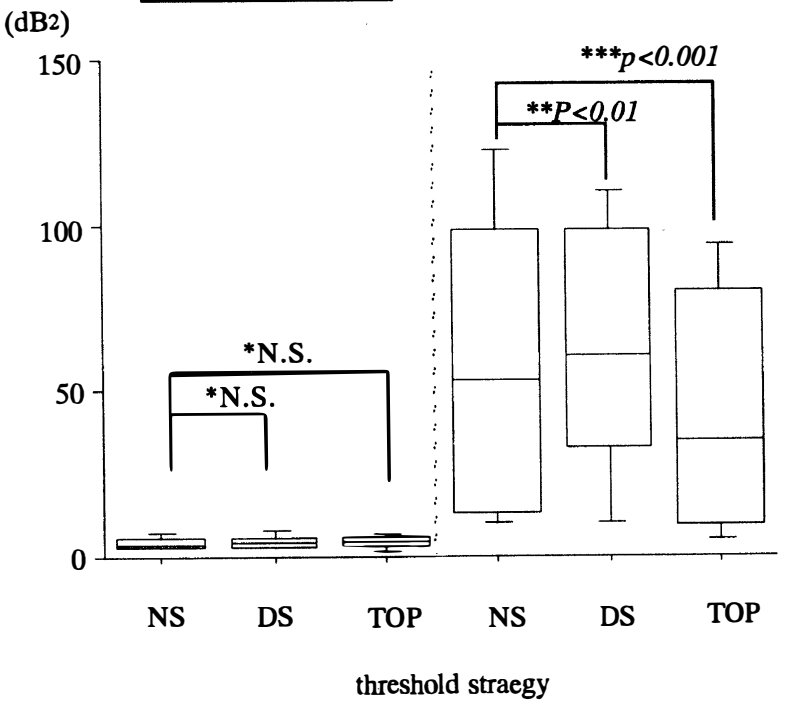

Fig. 3. The distribution of loss variance ( $L V)$ values in the control group and the glaucoma group. N.S., not significant. 
Table 4. The specificity and sensitivity of the DS and TOP strategy

\begin{tabular}{lcccc}
\hline & \multicolumn{2}{c}{ DS } & \multicolumn{2}{c}{ TOP } \\
\hline Specificity (\%) & $40 / 41$ & $(97.6 \%)$ & $37 / 41$ & $(90.2 \%)$ \\
Sensitivity (\%) & $32 / 36$ & $(88.9 \%)$ & $29 / 36$ & $(80.6 \%)$ \\
Stage I $^{\mathrm{a}}$ & $15 / 18$ & $(83.3 \%)$ & $13 / 18$ & $(72.2 \%)$ \\
Stage II $^{\mathrm{a}}$ & $9 / 10(90.0 \%)$ & $8 / 10$ & $(80.0 \%)$ \\
Stage III $^{\mathrm{a}}$ & $8 / 8$ & $(100.0 \%)$ & $8 / 8$ & $(100.0 \%)$ \\
\hline
\end{tabular}

DS, dynamic strategy; TOP, tendency oriented perimetry.

${ }^{a}$ According to the Aulhorn-Greves classification.

The measurement time using the DS was about $50 \%$ of that using the NS, and the time using the TOP strategy was slightly less than $20 \%$ of the NS. In patients with advanced visual field impairment, the percentage tiime saved was increased in the DS relative to the saving realised in patients having less severe loss. Previous studies ${ }^{10}$ have also shown time reductions of $43-46 \%$ using the DS and 79\% using the TOP strategy. Our results were similar to these rates. Concerning the global indices, the MS value did not differ among the three strategies in the control group or the glaucoma group. However, the LV value was lower using the TOP strategy than using the other two strategies. This suggests that the TOP strategy underestimates local visual field defects compared with the other two strategies. Lachkar et al. ${ }^{10}$ found no significant differences in the mean defect (MD), MS or short-term fluctuations (SF) as global indices between the TOP and NS strategies but observed a significantly lower LV for the TOP strategy than for the NS $(p<0.001)$ in glaucomatous eyes. Successful detection of local relative scotoma observed in the early stage of glaucoma might be affected by the sensitivity of neighbouring points due to the principles of the TOP strategy, and determination of the threshold may yield $\mathrm{LV}$ values lower than the actual value, resulting in underestimation of the range of the visual field defect.

The sensitivity and specificity of the DS were $88.9 \%$ and $97.6 \%$ (Table 4), respectively, which were comparable with those of the NS. Using the DS, measurement could be performed in a short time, especially in patients with advanced visual field loss. Therefore, the DS is appropriate for observation of the course in advanced cases. On the other hand, the sensitivity and specificity of the TOP strategy were $80.6 \%$ and $90.2 \%$, respectively, which were lower than those of the NS or DS. This strategy is likely to miss solitary relative scotoma as observed in the early stage of glaucoma, and measures such as addition of measurement points or use of programs exclusively for glaucoma such as $\mathrm{G} 1 \mathrm{X}^{24}$ for the grid are necessary. The low specificity of the $\mathrm{TOP}^{9}$ strategy may be because measurement at each of the 76 points was done only once, and incorrect responses of the subject may have affected adjacent points, and normal responses may have been misjudged as abnormal in some patients.

The detection rate of early stage glaucomatous visual field defects was lower for the DS and TOP strategy than for the NS. The TOP strategy allowed screening in a short time but the sensitivity and specificity were low, and caution is necessary in interpreting the results. Therefore, the TOP strategy may be appropriate for detection of patients in whom time-consuming perimetry is difficult, or the visual field defect is already advanced. Unlike strict threshold measurement methods, the TOP strategy ${ }^{11}$ uses estimation of the threshold from visual sensitivity at neighbouring points. Therefore, whether fluctuations in the threshold ${ }^{26}$ are caused by decreased sensitivity due to the disease itself $f^{25}$ or scattering due to estimated values $^{27}$ is difficult to determine when longterm observation of the course is necessary in diseases such as glaucoma. The long-term fluctuations of these strategies should be also evaluated. One study ${ }^{10}$ on the DS showed no significant test-to-test differences, but there have been no studies on the TOP strategy. Though the SF value was not evaluated in this study, Zulauf et al. ${ }^{7}$ observed a $23 \%$ increase in SF in the same subjects using the DS. It is possible that these strategies cause marked test-to-test fluctuations, which necessitates further studies.

Moreover, the DS and TOP strategy unlike the SITA, are also applicable to diseases other than glaucoma. In patients with advanced visual field defect in whom perimetry requires much time using the conventional NS, DS and TOP strategies may be useful. Finally, we would like to emphasise that the DS uses the fullthreshold method at sites of almost normal sensitivity, which also allows precise measurement of retinal sensitivity. Further studies and improvement of this strategy are needed.

\section{References}

1. Flammer J, Drance SM, Augustiny L, Funkhouser A. Quantification of glaucomatous visual field defects with automated perimetry. Invest Ophthalmol Vis Sci 1985;26:176-81.

2. Trope GE, Britton R. A comparison of Goldmann and Humphrey automated perimetry in patients with glaucoma. Br J Ophthalmol 1987;71:489-93.

3. Beck RW, Bergstrom TJ, Lichter PR. A clinical comparison of visual field testing with a new automated perimeter, the Humphrey Field Analyzer, and the Goldmann perimeter. Ophthalmology 1985;92:77-82.

4. Gillies B, Rivers M, Brooks AM. Comparison of Fastpak with full-threshold 24-2 glaucoma field tests. Ophthalmic Surg Lasers 1996;27:758-62.

5. Stewart WC, Shields MB, Ollie AR. Full threshold versus quantification of defects for visual field testing in glaucoma. Graefes Arch Clin Exp Ophthalmol 1989;227:51-4.

6. Hudson C, Wild JM, O'Neil EC. Fatigue effect during a single session of automated static threshold perimetry. Invest Ophthalmol Vis Sci 1994;35:268-80.

7. Zulauf M, Fehlmann P, Flammer J. Perimetry with normal Octopus technique and Weber 'dynamic' technique: initial results with reference to reproducibility of measurements in glaucoma patients. Ophthalmologe 1996;93:420-7.

8. Cho KW, Liu X, Loizou G, Wu JX. An AI approach to dynamic visual field testing. Comput Biomed Res 1998;31:143-63.

9. Weber J, Klimaschka T. Test time and efficiency of the dynamic strategy in glaucoma perimetry. Ger J Ophthalmol 1995;4:25-31.

10. Lachkar Y, Barrault O, Lefrancois A, Demailly P. Rapid tendency oriented perimeter (TOP) with the Octopus visual field analyzer. J Fr Ophtalmol 1998;21:180-4. 
11. de la Rosa MG, Marlinez A, Sanchez M, Mesa C, Cordovers L, Losada MJ. Accuracy of the tendency oriented perimetry (TOP) in the Octopus 1-2-3 perimetry. In: Wall M, editor. Perimetry update 1996/1997. Amsterdam: Kugler and Ghedini, 1998.

12. Aulhorn E. Pathophysiology of glaucomatous visual field defects. Fortschr Ophthalmol 1988;85:601-6.

13. Werner EB, Adelson A, Krupin T. Effect of patient experience on the results of automated perimetry in clinically stable glaucoma patients. Ophthalmology 1988;95:764-7.

14. Zulauf M, Caprioli J, Boeglin RJ, Lee M. Number of stimuli as a reliability parameter in perimetry. Ger J Ophthalmol 1992;1:86-90.

15. de Natale R, Glaab-Schrems E, Krieglstein GK. The prognosis of glaucoma investigated with computerized perimetry. Doc Ophthalmol 1984;58:385-92.

16. Hills JF, Johnson CA. Evaluation of the $t$ test as a method of detecting visual field changes. Ophthalmology 1988;95:261-6.

17. Johnson CA, Adams AJ, Casson EJ, Brandt JD. Blue-onyellow perimetry can predict the development of glaucomatous visual field loss. Arch Ophthalmol 1993;111:645-50.

18. Schenone M, Traverso CE, Molfino F, Capris P, Corallo G, Semino E. Comparison between computerized static perimetry and high-pass resolution perimetry in the followup of glaucomatous patients. Acta Ophthalmol Scand 1997;224:48-9.
19. Johnson CA, Samuels JS. Screening of glaucomatous visual field loss with frequency-doubling perimetry. Invest Ophthalmol Vis Sci 1997;38:413-25.

20. Bengtsson B, Olsson J, Heijl A, Rootzen H. A new generation of algorithms for computerized threshold perimetry, SITA. Acta Ophthalmol Scand 1997;75:368-75.

21. Bengtsson B, Heijl A, Olsson J. Evaluation of a new threshold visual field strategy, SITA, in normal subjects. Acta Ophthalmol Scand 1998;76:165-9.

22. Bengtsson B, Heijl A. Evaluation of a new perimetric strategy, SITA, in patients with manifest and suspect glaucoma. Acta Ophthalmol Scand 1998;76:268-72.

23. Bengtsson B, Heijl A. SITA Fast, a new perimetric threshold test: description of methods and evaluation in patients with manifest and suspect glaucoma. Acta Ophthalmol Scand 1998;76:431-7.

24. Messmer C, Flammer J. Octopus G1X. Ophthalmologica 1991;203:184-8.

25. Gloor BP, Vokt BA. Long-term fluctuation versus actual field loss in glaucoma patients. Dev Ophthalmol 1985;12:48-69.

26. Dengler-Harles M, Wild JM, Cole MD, O'Neil EC. The influence of stimulus parameters on the visual field indices by automated projection perimetry. Graefes Arch Clin Exp Ophthalmol 1993;231:146-50.

27. Spenceley SE, Henson DB. Visual field test simulation and error in threshold estimation. Br J Ophthalmol 1996;80:304-8. 